\title{
Téoros
}

Revue de recherche en tourisme

\section{Un vieux débat toujours ranimé autour de l'interprétation du patrimoine}

\section{Martin Drouin}

Volume 26, numéro 3, automne 2007

URI : https://id.erudit.org/iderudit/1071010ar

DOI : https://doi.org/10.7202/1071010ar

Aller au sommaire du numéro

Éditeur(s)

Université du Québec à Montréal

ISSN

0712-8657 (imprimé)

1923-2705 (numérique)

Découvrir la revue

Citer cette note

Drouin, M. (2007). Un vieux débat toujours ranimé autour de l'interprétation du patrimoine. Téoros, 26(3), 72-75. https://doi.org/10.7202/1071010ar d'utilisation que vous pouvez consulter en ligne.

https://apropos.erudit.org/fr/usagers/politique-dutilisation/ 
$\Rightarrow$

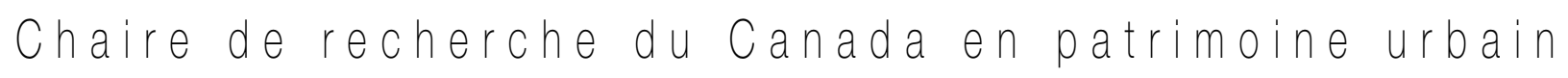

\section{Un vieux débat toujours ranimé autour de l'interprétation du patrimoine}

\section{Martin Drouin}

Nombreux sont ceux et celles qui ont pris la route des vacances cet été. La douce oisiveté ayant ses limites, plus d'un ont choisi de visiter un site historique, un centre d'interprétation ou un musée. La fréquentation du patrimoine constitue désormais, on le sait, une ressource incontournable dans le choix des activités de loisir. Que ce soit pour le divertissement, par intérêt, pour éviter la pluie ou faire plaisir à sa conjointe ou son conjoint, les raisons sont multiples et ne cadrent pas toujours avec les nobles déclinaisons du tourisme culturel. Qu'importe! Le visiteur vivra une expérience muséale qui le confrontera à diverses formes d'interprétation. Si, par la thématique du lieu, il a déjà une idée de ce qu'il verra, il ne sait pas, en général, ce qui l'attend. L'aventure commence par et à l'accueil. Un premier contact lui permettra d'obtenir quelques renseignements et de s'acquitter du droit d'entrée. Se poseront alors les premiers dilemmes. Payer un supplément pour l'audioguide? Suivre une visite guidée? Prendre la route en s'en remettant à l'interprétation disponible à l'intérieur des salles ou sur le site? Dans tous les cas, il devra répondre rapidement. À moins que la réponse ne lui soit imposée par les contraintes du lieu. Quoiqu'il en soit, le voilà néanmoins confronté à la « lourde » décision de choisir entre la fréquentation de la technologie ou de l'être humain.

Ce débat n'a rien d'orwelien. II n'est pas nouveau non plus. Pour John Muir (White, 2006), Enos Mills (1920) ou Freeman Tilden (1957), les pionniers de l'interprétation, le guide détient tous les atouts et les pouvoirs pour livrer les secrets d'un site. Ceux-ci ne se doutaient pas, à l'époque, des possibilités futures. Au fur et à mesure du développement et de la démocratisation des technologies, d'aucuns y ont vu une chance inestimable. La machine permettait enfin de dépasser les contingences humaines. La diffusion s'est faite d'autant plus rapidement que la promesse d'une nouvelle manière de procéder correspondait à l'essor des centres d'interprétation et à l'investissement de sommes encore considérables dans les efforts muséographiques. C'est à partir de ce moment, et des dérapages qui s'en sont suivis, que deux camps se sont formés. Certains ont aimé, se sont amusés et émerveillés. D'autres ont accueilli avec scepticisme et déception la muséologie «à bouton ». Au fil des sites, le visiteur qui répétera l'expérience - par plaisir, espérons-le, à moins que la température n'ait été vraiment mauvaise - développera lui aussi son goût. Son cheminement et son tempérament le feront pencher vers l'une ou l'autre des façons de procéder. II rejettera carrément certaines pratiques, tandis qu'il acceptera avec soulagement tel autre type d'interprétation. La question refait immanquablement surface.

Ce ne sont pas les débats qui ont arrêté la technologie dans son développement au service de l'interprétation du patrimoine. Au contraire, elle s'est même institutionnalisée. Depuis une dizaine d'années, par exemple, l'Ename Center for Public Archaeology and Heritage Presentation est devenu un pôle majeur de recherche ([www.enamecenter.org]). Ce centre découle du projet « Ename 974 » qui avait été lancé dans les années 1980¹. Basé en Belgique flamande, il s'est depuis donné pour mission d'améliorer l'emploi 
des nouvelles technologies de l'information dans des lieux patrimoniaux. Sa principale réalisation est le système TimeScope : implanté sur des sites archéologiques, un kiosque équipé de caméras numériques, d'un ordinateur et d'écrans tactiles offre une occasion unique au visiteur de visualiser des constructions anciennes. L'utilisation d'images captées en temps réel et l'incrustation de modèles 3D permettent de maximiser l'illusion de réalité de lieux aujourd'hui disparus. Plus récemment, une alliance de recherche européenne - EPOCH${ }^{2}$ - a vu le jour avec l'objectif de poursuivre et de multiplier les réflexions et les initiatives de ce type ([www. epoch-net.org]). Elle s'inscrit dans la dynamique de partage des symposiums VAST (Virtual Reality, Archeology and Intelligent Cultural Heritage $)^{3}$. De nouveaux professionnels sont ainsi en train de prendre leur place aux côtés des archéologues, des architectes, des ethnologues et des historiens. La technologie sera - et c'est un truisme - de plus en plus présente dans l'interprétation du patrimoine.

II n'est pas étonnant que le centre Ename héberge aujourd'hui le secrétariat du comité scientifique du Conseil international des monuments et des sites (ICOMOS) sur l'Interprétation et la présentation des sites culturels patrimoniaux (ICIP). Créé il y a quelques années, le comité a d'ailleurs mis sur pied un groupe de travail sur les technologies interprétatives émergentes. Sa mission est de favoriser le dialogue entre les spécialistes des technologies et les professionnels du patrimoine afin de «supporter et encourager le déploiement à la fois des nouvelles technologies et de nouvelles utilisations de celles déjà existantes au niveau de l'interprétation et la présentation de sites dans le monde entier » ([http://icip.icomos.org]). L'initiative la plus diffusée du comité est cependant la rédaction d'une charte pour l'interprétation et la présentation des sites culturels patrimoniaux, d'abord connue sous le nom de Charte d'Ename, puis de Déclaration de Charleston ${ }^{4}$. L'objectif de la charte est de baliser les pratiques pour éviter les dérapages « quant aux finalités de la conservation et l'appréciation du public des sites patrimoniaux 》 (ICIP-ICOMOS, 2007 : 3). Sept principes ont ainsi été énoncés ${ }^{5}$. Le plus étonnant est que le document traite très peu de l'utilisation des nouvelles technologies de l'information, même si la rédaction avait été intimement liée au centre Ename. N'y aurait-il que des lendemains qui chantent pour les formes émergentes de l'interprétation du patrimoine? La technologie n'influe-t-elle pas sur la manière d'interpréter? Le véhicule n'est pourtant pas neutre.

De nombreux problèmes ont déjà été notés. Ils sont de plusieurs ordres, que ce soit par rapport à l'investissement, à la fiabilité, à l'impact sur les visiteurs ou sur le contenu de l'exposition. En voici quelques-uns. Le coût des technologies, d'abord, est souvent écrasant lors de l'installation. De plus, l'entretien des appareils grève les budgets de fonctionnement en plus de nécessiter l'emploi de personnels qualifiés sinon affectés à d'autres tâches. Des ratés techniques sont plus facilement susceptibles de transformer négativement l'expérience du touriste, ce qui est en contradiction fondamentale avec les objectifs de l'interprétation. Le nombre de visiteurs rend fréquemment la manipulation et la fréquentation de modules plus difficiles. Les multimédias excitent d'ordinaire des groupes potentiellement turbulents et perturbent conséquemment la quiétude de certains lieux. Les sons provenant de postes de consultation peuvent aussi troubler les visites dans une ambiance proche de la cacophonie (Neill, $2003:$ 7).

Avec l'utilisation de technologies, les visiteurs se trouvent souvent dans une position passive de réception et sont ainsi limités dans leur interaction et leur dialogue au cours de l'exposition. L'interprétation utilisant les nouvelles technologies de l'information est par définition statique tout au long de sa durée de vie; elle ne peut évoluer, voire se bonifier en cours d'utilisation. Plus encore, la réalité virtuelle se confond avec l'impression d'une vérité historique. Or, à cette enseigne, la discipline historique pose généralement des bémols face à un passé rempli d'incertitudes. L'emploi des technologies commande cependant une profusion de données d'une exactitude toujours plus grande ; la conception doit dès lors s'appuyer sur des conjectures dont l'ampleur est difficilement communicable. Neil Silberman, directeur du centre Ename et du Comité scientifique international sur l'interprétation et la présentation des sites patrimoniaux,

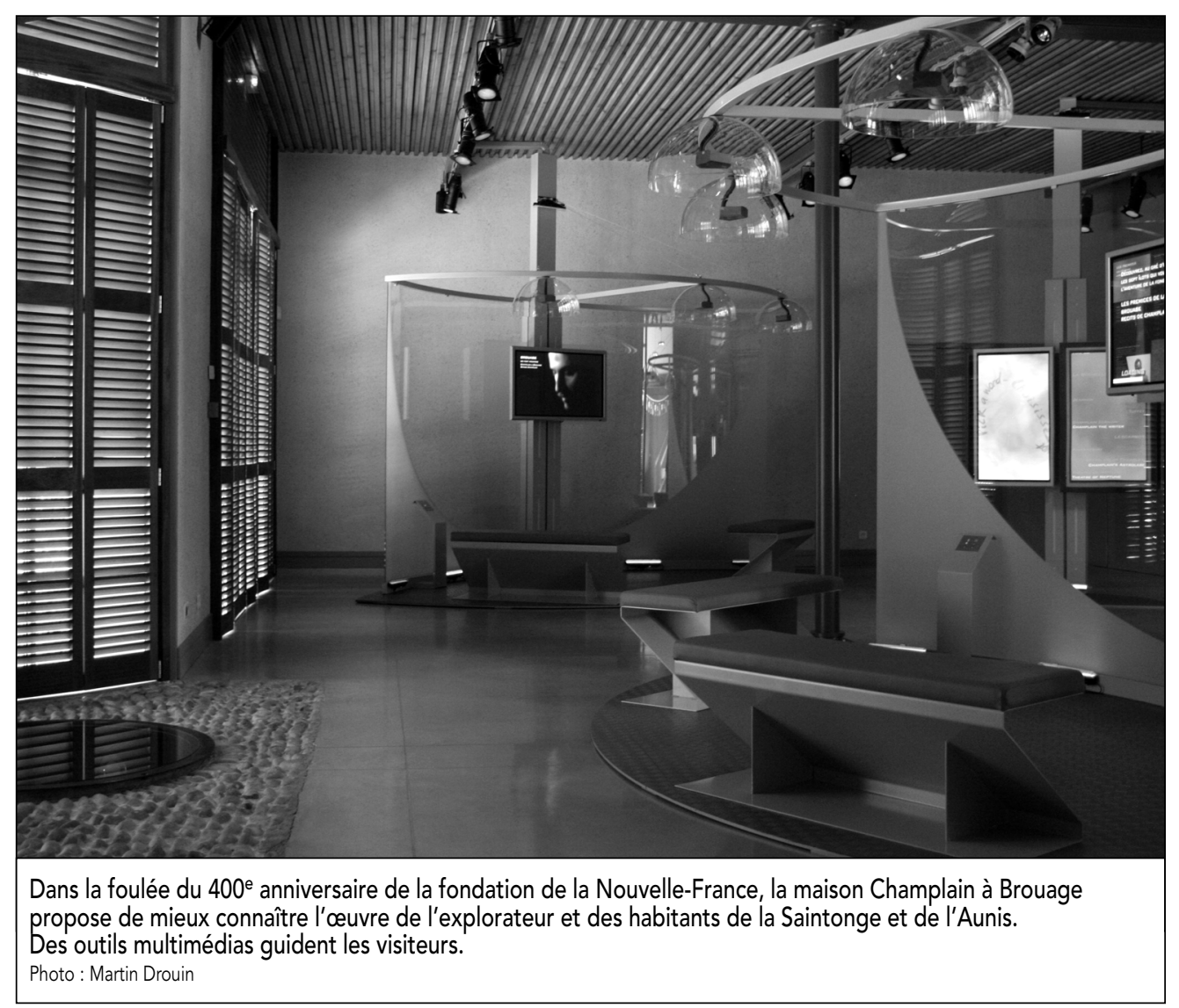


soulignait à ce propos les dangers de confusion entre le virtuel et la perception du passé, soulevant ainsi tout le problème de l'authenticité des matériaux utilisés (Liuzza, 2006). Dans des situations extrêmes, l'expérience muséale est complètement déconnectée du milieu à interpréter. À Brouage, à la maison de Champlain notamment, le visiteur transite à travers une série de modules vidéo qui présentent différentes capsules informatives. Même s'il se trouve dans la ville natale du célèbre explorateur, il perd la relation à l'environnement - d'ailleurs tout à fait exceptionnel - qui devrait faire partie du programme d'interprétation. ॥ est dans ces conditions difficile de s'appuyer exclusivement sur les formes émergentes de l'interprétation du patrimoine.

II serait injuste de brosser un tableau complètement noir de la situation. Des expériences et des sites font en effet rêver. À trente minutes de la capitale écossaise, à North Berwick sur le littoral de la mer du Nord, le Scottish Seabird Center se consacre à l'initiation et à la protection du patrimoine naturel. Gagnant de plusieurs prix, remis par des organismes environnementaux ou de promotion du tourisme, le centre de découverte s'intéresse à la vie animale. L'aspect le plus spectaculaire de la visite consiste à manipuler, à l'aide d'une commande à distance, une caméra installée non loin dans les îles. En tout, quatre appareils permettent d'observer le quotidien des oiseaux de mer sans déranger leur milieu de vie. L'utilisation s'inscrit ici dans la volonté de proposer un tourisme durable ([www.seabird.org]). Le guide multimédia mobile offre, quant à lui, des possibilités tout à fait exceptionnelles. Testé sur les sites archéologiques de Pompéi en Italie et d'Olympe en Grèce, l'appareil donne l'occasion aux visiteurs de visualiser, par le truchement de lunettes vidéo, ces villes à leurs plus belles heures de gloire. L'emploi

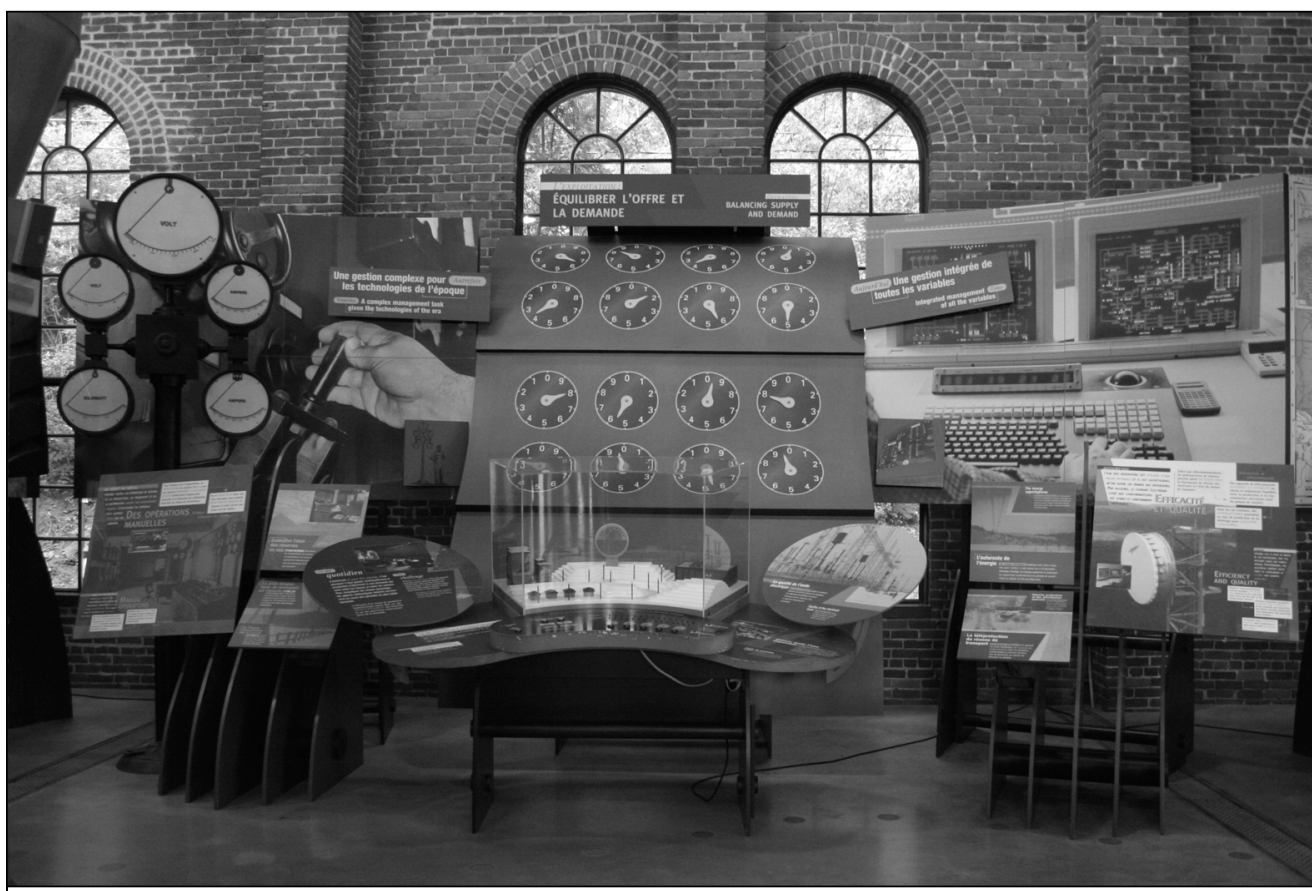

À la Cité de l'énergie, un important dispositif muséographique a été installé, tant dans le centre des sciences que dans le secteur historique. Le visiteur peut s'initier à la fabrication de l'électricité et du papier à l'aide de différentes expériences interactives.

Photo: Marie-Blanche Fourcade d'un système de positionnement par satellite localise l'utilisateur en temps réel (Owen et al., 2006 : 390). À Montréal, à Pointe-à-Callière, le musée d'archéologie et d'histoire fait une utilisation très judicieuse du procédé holographique dans son exposition permanente. Les liens entre le passé et le présent sont ainsi suggérés dans une atmosphère éthérée. Le papier électronique, les présentoirs à surface adaptable, l'identification par radiofréquence et bien d'autres technologies pourraient révolutionner l'interprétation du patrimoine, tout comme le téléchargement de visites en baladodiffusion a transformé certaines formes du tourisme urbain.

La technologie est là. Toujours remplie de promesses. Pourquoi se questionne-t-on encore à choisir entre les nouvelles technologies de l'information et l'interprète? Parce que ce dernier possède encore toute sa pertinence. Le responsable d'un site rencontré récemment l'exprimait avec inquiétude. Le sous-financement l'avait obligé à ne pas réengager les employés de la saison estivale, car il n'avait pu couper dans l'entretien des lieux. II se devait avant tout de veiller à la conservation de la ressource. Si l'endroit porte toujours en lui-même un pouvoir d'enseignement exceptionnel, il ne peut se révéler seul. Dans bien des situations, l'émotion se transmet plus facilement grâce au travail du guide. La narration d'une légende amérindienne par une interprète du site de la Nouvelle-France, à Saint-Félix d'Otis dans le Bas-Saguenay, le rappelle à ceux qui l'ont entendue. Le son du tambour qui rythmait son intrigue pour culminer avec le chant du huard qu'elle imitait à la perfection laissait l'assemblée stupéfaite. Tous étaient en train de vivre un moment unique. L'infime parcelle d'un monde disparu venait d'être transmise. À n'en pas douter, le guide a des qualités indéniables. II s'adapte, écoute, dialogue et interagit. L'expérience personnelle et professionnelle de l'interprète se relaie et se bonifie d'ailleurs au contact de celle des visiteurs. Ce mineur qui faisait visiter l'ancien carreau de la mine du bois du Cazier à Marcinelle en Belgique communiquait une connaissance unique des lieux et des pratiques, multipliant ainsi la force évocatrice du site. Alors que je travaillais pour Parcs Canada au Lieu historique national du Coteau-du-Lac, au sud-ouest de Montréal, bien des visiteurs ont partagé leur souvenir ou leur savoir au bénéfice du groupe. II n'était pas rare à ce moment-là que d'autres renchérissent. Nous étions alors tous ensemble portés par la vie qui avait été vécue sur ces lieux et par les trajectoires de chacun. L'information pouvait, bien sûr, être ensuite utilisée dans une présentation ultérieure. Le visiteur n'est pas passif et la visite n'est pas statique. Comme l'écrivait Enos Mills dans son ouvrage The Aventures of a Nature Guide, "The essence of [interpretation] is to travel gracefully rather than to arrive. " (Mills, 1920 : 125, cité par Beck et Gable, 2002 : 169) Aurait-on l'impression que ce voyage serait davantage possible dans la rencontre d'autrui ? 
À n'en pas douter, ces multiples raisons expliquent que le débat existe encore. À l'inverse, on en discute aussi parce que la relation avec un guide n'est pas toujours facile et que la technologie peut incarner une certaine fiabilité vis-à-vis de l'individu. Freeman Tilden (1957) l'affirmait en des termes clairs : l'interprétation est un art. Devant ce constat, des expériences peuvent être désastreuses. L'un récite son texte par cœur-comme une machine diront les mauvaises langues -, l'autre pontifie du haut de son savoir; l'inexpérience d'un troisième lui fait prononcer quelques énormités. Face à cela, la technologie assure une stabilité du contenu. La gestion de la qualité est ainsi validée par des professionnels, tout comme la pertinence d'activités ludiques ou pédagogiques. L'intégration d'images, de cartes, de graphiques, d'objets, de chansons, de musiques ou d'ambiances sonores rend bien pâle un récit monocorde à la voix nasillarde. L'interprétation, souligne le même Tilden, est aussi une technique qui s'apprend. L'interprète est comme le bon vin, alors que la machine a rapidement des airs de désuétude et d'obsolescence. Comment dès lors choisir entre I'utilisation de nouvelles technologies de l'information et des individus ? Le coût d'investissement initial ne départagerait-il pas finalement les utilisateurs de technologie? Pour une exposition permanente à la Cité de l'énergie de Shawinigan, il y a bien des sites touristiques qui doivent miser uniquement sur la passion et la connaissance des guides, n'ayant pas les moyens de faire autrement. Bref, il serait possible de raviver encore longtemps ce vieux débat autour de l'interprétation du patrimoine.

Des esprits malins suggéreront de conjuguer les deux avenues afin de bonifier l'ensemble de l'expérience. Dans cette perspective, j'ai été bien étonné de voir une interprète de la Corderie royale de Rochefort expliquer l'histoire du bâtiment accompagnée d'une projection multimédia. Le musée avait ainsi pris le parti de ne pas enregistrer la voix de la narratrice. L'interprétation d'un site est un exercice délicat et difficile; il faut dès lors s'assurer de mettre toutes les chances de son côté. La question ne serait-elle cependant pas ailleurs ? Ne faudrait-il pas plutôt se questionner sur la pertinence de l'interprétation vis-à-vis de la ressource ? C'est pour cette dernière finalement que le visiteur se déplace. Les expositions temporaires et autres attractions multimédias ne sont - et il ne faut pas l'oublier - qu'une façon de réinscrire l'intérêt d'un site. Spectacle à grand déploiement ou guide passionnant, le visiteur doit surtout se demander s'il a été interpellé. Est-ce que le lieu interprété lui a été révélé par les méthodes employées ? C'est finalement le patrimoine qui doit être au centre du débat. On ne se pose d'ailleurs peut-être pas assez la question...

Martin Drouin est professeur associé au Département d'études urbaines et touristique de l'Université du Québec à Montréal.

\section{Notes}

1 La ville d'Ename possède une histoire millénaire que des vestiges archéologiques permettent d'interpréter. Le projet «Ename 974 » a consisté à développer différentes techniques afin de communiquer cette histoire à un grand public. Outre le parc archéologique de huit hectares, l'église Saint-Laurentien, le boisé Bos t'Ename et un musée sont aujourd'hui ouverts aux visiteurs.

2 European Research Network of Excellence in Open Cultural Heritage.
3 Une huitième rencontre, intitulée Future Technologies To Empower Heritage Professionals, aura lieu au mois de novembre 2007 à Brighton, au Royaume-Uni. (À ce sujet, voir VAST, 2007.)

4 La charte devrait être approuvée lors de l'assemblée générale qui se tiendra à Québec en 2008. (Voir ICOMOS Ename Charter for the Interpretation of Cultural Heritage Sites, 2007.)

5 1) accès et compréhension, 2) solidité des sources d'information, 3) attention portée au contexte et à l'environnement, 4) préservation de l'authenticité, 5) organisation de la durabilité, 6) attention portée à la participation, 7) importance de la recherche, de la formation et de l'évaluation.

\section{Bibliographie}

Beck, Larry, et Ted Gable (2002), Interpretation for the $21^{\text {st }}$ Century. Fifteen Guiding Principles for Interpreting Nature and Culture, Champagne, Illinois, Sagamore Publishing.

Ename 974 (n.d.), [www.ename974.org], (consulté le 17 août 2007).

Ename Center for Public Archaeology and Heritage Presentation (2007), [www.enamecenter.org], (consulté le 17 août 2007).

EPOCH (2002-2007), [www.epoch-net.org], (consulté le 17 août 2007).

ICIP-ICOMOS (n.d.), [http://icip.icomos.org], (consulté le 17 août 2007).

ICIP-ICOMOS (2007), "Charte ICOMOS pour l'interprétation et la présentation des sites culturels patrimoniaux ", [http://icip.icomos.org/FR/ groups_charter.html], (consulté le 17 août 2007).

ICOMOS Ename Charter for the Interpretation of Cultural Heritage Sites (2007), [http://www.enamecharter.org/], (consulté le 17 août 2007).

Liuzza, Claude (2006), «Compte-rendu de la table ronde d'une durée d'un jour: normes d'interprétation pour les sites patrimoniaux mondiaux: Existe-t-il un besoin de répartition des critères de programmes d'interprétation in situ et d'émergence de technologies interprétatives? ", Comité scientifique international d'ICOMOS sur l'interprétation et la présentation des sites patrimoniaux, [http://icip.icomos.org/FR/agen da_meeting27092006.html], (consulté le 17 août 2007).

Mills, Enos (1920) [rééd. 1990], The Adventures of a Nature Guide and Essays on Interpretation, Friendship, Wisconsin, New Past Press.

Neill, George (2003), "Working with Multimedia », Interpret Scotland, the Journal for Scotland's Interpreters, $n^{\circ} 7$, printemps, p. 7.

Owen, Ruth, Dimitrios Buhalis, et Daniël Pletinckx (2006), « Visitors' Evaluations of Technology Used at Cultural Heritage Sites ", Information and Communication Technologies in Tourism 2006, Proceedings of the International Conference in Lausanne, Switzerland, Vienne, Springer, p. 383-393.

Tilden, Freeman (1957) [3éd. 1977], Interpreting our Heritage, Chapel Hill, University of North Carolina.

VAST (2007), [http://aranea.brighton.ac.uk/vast2007/], (consulté le 17 août 2007).

White, Fred, dir. (2006), Essential Muir: A Selection of John Muir's Best Writings, Berkeley (Californie), Heyday Books. 